\title{
REPLACING HOMOTOPY ACTIONS BY TOPOLOGICAL ACTIONS
}

BY

GEORGE COOKE

\begin{abstract}
A homotopy action of a group $G$ on a space $X$ is a homomorphism from $G$ to the group of homotopy classes of homotopy equivalences of $X$. The question studied in this paper is: When is a homotopy action equivalent, in an appropriate sense, to a topological action of $G$ on $X$ ?
\end{abstract}

Introduction. A homotopy action of a group $G$ on a space $X$ is a homomorphism $\alpha$ from $G$ to the group $\mathcal{E}(X)$ of homotopy classes of homotopy equivalences of $X$. If $G$ acts on $X$ via homeomorphisms, the associated homotopy action is called topological. A homotopy action $\alpha$ of $G$ on $X$ is equivalent to a homotopy action $\beta$ of $G$ on $Y$ if there exists a homotopy equivalence $f: X \rightarrow Y$ such that the diagram

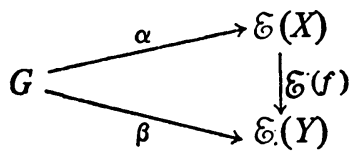

is commutative. Here $\mathcal{E}(f)$ is defined by $[g] \mapsto\left[f g f^{-1}\right]$, where $[g] \in \mathcal{E}(X)$ and $f^{-1}$ is any homotopy inverse for $f$.

The purpose of this paper is to study the following question and to derive some applications from the answer: Under what conditions is a homotopy action of $G$ on $X$ equivalent to a topological action of $G$ on some other space $Y$ ?

The first result of this paper gives a homotopy-theoretic treatment of the problem (Theorem 1.1 below).

Let $X$ be a space having the homotopy type of a CW complex. (All spaces will satisfy this restriction from now on.) Let $G(X)$ denote the space of self-homotopy equivalences of $X$. It is an associative $H$-space satisfying $\pi_{0} G(X)=\mathcal{E}(X)$. The identity component is denoted $G_{1}(X)$, and there is an

Received by the editors November 17, 1976. George Cooke died in December 1976, before the referee's report could be sent to him. At the request of the editor (Church) and in consultation with Kenneth Brown, Peter Kahn revised the manuscript somewhat; he balanced well the conflicting claims of fidelity to the author's manuscript and the need for some revision the author could no longer make. The editor is very grateful to them.

AMS (MOS) subject classifications (1970). Primary 55D10, 55D20; Secondary 55G35, 55E99. 
exact sequence of $H$-spaces

$$
G_{1}(X) \rightarrow G(X) \stackrel{\pi}{\rightarrow} \pi_{0}(G(X))=\mathcal{E}(X) .
$$

The map $\pi$ on (0.1) induces a map of classifying spaces (in the sense of Stasheff [7]; see also Allaud [2])

$$
B_{G(X)} \stackrel{B_{\pi}}{\rightarrow} K(\mathcal{E}(X), 1) .
$$

The fibre of $B_{\pi}$ is homotopy equivalent to $B_{G_{1}(X)}$.

Associated to the homotopy action $\alpha$ of $G$ on $X$ is then a lifting problem

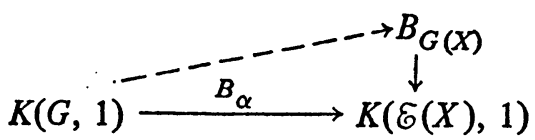

Since $\pi_{1} B_{G(X)} \rightarrow \pi_{1} K(\mathcal{E}(X), 1)$ is an isomorphism, there is a lifting over the 2-skeleton of $K(G, 1)$.

THEOREM 1.1. (a) The homotopy action $\alpha$ is equivalent to a topological action of $G$ on some space $Y$ if and only if the lifting problem (0.2) has an affirmative solution. Therefore:

(b) There is an obstruction theory for the problem of replacing $\alpha$ by an equivalent topological action, with obstructions lying in the groups

$$
H^{n}\left(G,\left\{\pi_{n-2}\left(G_{1}(X)\right)\right\}\right), \quad n>3 .
$$

In particular, if the groups (0.3) vanish, then $\alpha$ is equivalent to a topological action.

This theorem is proved in $\S 1$. As an immediate consequence, one has the following:

COROLLARY. If $G$ is free, or, more generally, if there exists a 2-dimensional $K(G, 1)$, then a homotopy action of $G$ can always be replaced by a topological action.

The case $G$ free has a more direct proof which we describe in $\S 1$.

For use in applications, we note that any topological action of $G$ on a space $X$ is equivalent to a free topological action of $G$ on a space $Y$ (i.e., $G$ acts via deck transformations on the covering space $Y$ of $Y / G)$. To see this, use any contractible, free $G$-space $W$, set $Y=X \times W$, and let $G$ act on $Y$ via the diagonal action.

The first application provides an affirmative answer to the following question, posed by John Moore in 1967: Does there exist a space $X$ and an element $\theta \in \pi_{1} X$ such that $\theta$ acts (via deck transformations) trivially on the homotopy and nontrivially on the homology of the universal cover $\tilde{X}$ ?

Using the above corollary (for the case $G=\mathbf{Z}$ ) and the remarks following 
it, one sees that Moore's question is equivalent to the following: Does there exist a 1-connected space $Y$ and a self-equivalence $f: Y \rightarrow Y$ such that $f_{*}$ : $\pi_{*} Y \rightarrow \pi_{*} Y$ is the identity and $f_{*}: H_{*} Y \rightarrow H_{*} Y$ is not? An example of such a $Y$ and $f$ is given in $\S 1$.

The philosophy behind this application, and subsequent ones, is the following: It is relatively easy to find homotopy actions of a group $G$ on a space $X$ with interesting homotopical and homological properties. One attempts then to use Theorem 1.1 and subsequent remarks to replace this action by an equivalent deck-transformation action of $G$ on $Y$. Assuming this step is successful, one obtains the quotient $Y / G$ whose homotopy and homology often reflect the interesting properties of the original homotopy action.

To carry out this program, it is useful to have a class of spaces $X$ and groups $G$ such that every homotopy action of $G$ on $X$ can be replaced by a topological action. Such a class is described in the next result (Theorem 2.3 below).

Let $h_{*}$ be any generalized homology theory. I use Bousfield's notion of $h_{*}$-local spaces and $h_{*}$-localization [3] (see $\$ 2$ ), and so I assume that $h_{*}$ satisfies the limit axiom [1, p. 188], which asserts that $h_{*}(X)$ is the direct limit of $h_{*}(K)$, where $K$ ranges over the finite subcomplexes of $X$.

THEOREM 2.3. Let $G$ be a group of order $n$, and suppose that multiplication by $n$ gives an isomorphism of $h_{*}(\mathrm{pt})$. Let $X$ be an $h_{*}$-local space. Then every homotopy action of $G$ on $X$ is equivalent to a topological action of $G$ on some space $Y$.

When Theorem 2.3 is applied to the Bousfield $h_{*}$-localization of an arbitrary space $X$, where $h_{*}=H_{*}(; R), R=\mathbf{Z} / p$ or $R \subseteq \mathbf{Q}$, the result is the following realizability theorem:

THEOREM 2.4. Let $X$ be any space, $G$ a group whose order is invertible in $R$, and $\alpha$ a homotopy action of $G$ on $X$. Then, there is a space $X_{\alpha}$ and a map $f$ : $X \rightarrow X_{\alpha}$ such that $f^{*}$ maps $h^{*}\left(X_{\alpha}\right)$ isomorphically onto $h^{*}(X)^{G}$, the cohomology of $X$ which is fixed by $G$.

For another application, I show that if $X$ is $h_{*}$-local, $h_{*}=H_{*}(; R)$ as above, and if $P$ is the set of primes not invertible in $R$, then the only torsion in $\mathcal{E}_{1}(X)$ is $P$-torsion (Theorem 2.5). Here, $\mathcal{E}_{1}(X) \subseteq \mathcal{E}(X)$ consists of all classes of homotopy equivalences inducing the identity on homology. This supports the conjecture that $\mathcal{E}_{1}(X)$ is $h_{*}$-local.

A further application, to be described in [5], is in the construction of spaces with interesting cohomological properties via group actions. This again follows the philosophy outlined above. To facilitate the construction in [5], I 
include in the appendix a couple of propositions on relative homotopy actions.

The foregoing applications suggest the problem of classifying, for fixed $G$ and 1-connected $X$, those homotopy types having universal cover homotopy equivalent to $X$ and fundamental group isomorphic to $G$. Such types correspond essentially to choices of lifting in the diagram (0.2). I give such a classification at the end of $\S 1$.

Finally, to show that the theory is nonvacuous, I give an example in $\S 3$ of a 1-connected space admitting a homotopy action (by $\mathrm{Z} / 2$ ) that cannot be replaced by a topological action.

I wish to thank Ken Brown for useful conversations about localization and homotopy theory, and, in particular, for bringing Bousfield's work [3] to my attention.

1. Replacing a homotopy action by a topological action. I begin with some remarks concerning the Stasheff classifying space. The space $B_{G(X)}$ is the base space of a fibration $\zeta$ with fibre having the homotopy type of $X$. Spaces have base points, and there is a fixed homotopy equivalence $f_{\xi}: X \rightarrow \zeta^{-1}(*)$.

An $X$-fibration over a space $B$ is a pair $\left(\theta, f_{\theta}\right)$, where $\theta: E \rightarrow B$ a fibration and $f_{\theta}: X \rightarrow \theta^{-1}(*)$ is a homotopy equivalence. According to Allaud [2], the $X$-fibration $\left(\zeta, f_{\zeta}\right)$ over $B_{G(X)}$ is universal, in the sense that given any $X$ fibration $\left(\theta, f_{\theta}\right)$ over $B$, there is a unique homotopy class $[f] \in\left[B, B_{G(X)}\right]$ such that $f^{*}(\zeta)$ is fibre homotopy equivalent to $\theta$, by a fibre homotopy equivalence compatible with the given homotopy equivalences of $X$ and fibres.

In the fibration $E \rightarrow B$, with fibre $F$, there is an associated homotopy action of $\pi_{1}(B)$ on $F$, and hence on $X$. This homotopy action

$$
\pi_{1}(B) \rightarrow \mathcal{E}(X)
$$

may be determined as the map on fundamental groups induced by the composition

$$
B \stackrel{f}{\rightarrow} B_{G(X)} \stackrel{B_{\pi}}{\rightarrow} K(\mathcal{E}(X), 1) .
$$

Proof OF THEOREM 1.1. Let $\alpha$ be a homotopy action of the group $G$ on $X$. Suppose that there is a homotopy equivalence $f: X \rightarrow Y$ and a compatible topological action $\beta$ of $G$ on $Y$. The action $\beta$ may be assumed fixed point free by crossing with a contractible free $G$-space and replacing $\beta$ by the diagonal action if necessary. Consider the covering map $Y \rightarrow Y / G$. This is induced from the universal covering of $K(G, 1)$ by a map

$$
Y / G \stackrel{\theta}{\rightarrow} K(G, 1) \text {. }
$$

Convert $\theta$ into a fibration $\bar{\theta}: E_{\theta} \rightarrow K(G, 1)$. There is a well-defined homotopy equivalence between $\bar{\theta}^{-1}(*)$ and the space $Y$, and, hence, via $f$, a well-defined 
homotopy equivalence $f_{\bar{\theta}}: X \rightarrow \bar{\theta}^{-1}(*)$. The associated homotopy action of $G$ on $\bar{\theta}^{-1}(*)$ is equivalent via $f_{\bar{\theta}}$ to the given action $\alpha$ of $G$ on $X$.

According to the remarks on $B_{G(X)}$, the fibration $\left(\bar{\theta}, f_{\bar{\theta}}\right)$ is induced by a map

$$
K(G, 1) \stackrel{\varphi}{\rightarrow} B_{G(X)}
$$

to the classifying space. The diagram

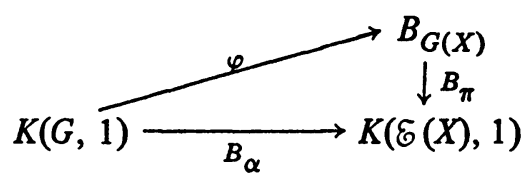

is commutative; it is enough to check the maps on $\pi_{1}$, and commutativity on $\pi_{1}$ is clear from the construction and the remarks preceding the beginning of the proof.

Conversely, let us assume that the lifting problem

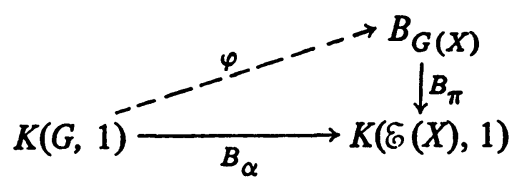

has a solution $\varphi$. Consider the pull-back $\varphi^{*}(\zeta)$. This is a fibration with fibre having the homotopy type of $X$. Denote the total space by $E$. Consider the pull-back of the universal cover

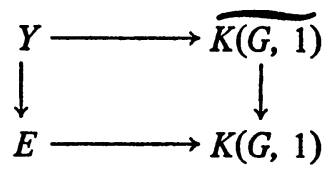

Then $Y$ has the homotopy type of $X, G$ acts on $Y$, and it is easy to see that this action is equivalent to the original action $\alpha$ of $G$ on $X$. This completes the proof of Theorem 1.1.

In case $G$ is free there is a more direct approach. We describe only the case $G=\mathbf{Z}$. To give a homotopy action by $G$ on a space $X$ is simply to specify a homotopy equivalence $h: X \rightarrow X$. Now let $Y$ denote the infinite mapping cylinder of $h$ :

$$
Y=(\mathrm{Z} \times I \times X) /(n, 1, x) \sim(n+1,0, h(x)) .
$$

Let $T: Y \rightarrow Y$ denote the shift: $T(n, t, x)=(n-1, t, x)$. Then $T$ generates a topological action by $\mathbf{Z}$ on $Y$. There is a homotopy equivalence $f: X \rightarrow Y$ defined by $f(x)=(0,0, x)$. The diagram 


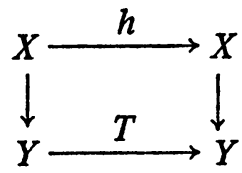

homotopy commutes, showing that the given homotopy action of $\mathbf{Z}$ on $X$ is equivalent to the topological action of $\mathbf{Z}$ on $Y$ defined by $T$. This means that the covering transformation $T$ is homotopically equivalent to the original homotopy equivalence $h: X \rightarrow X$ and shows that every homotopy equivalence can be realized, in the appropriate sense, as a covering transformation. A generalization of this infinite iterated mapping cylinder construction gives the analogous result for all free $G$.

This result implies an affirmative answer to the question of John Moore which was stated in the Introduction. It suffices to find a 1-connected space $X$ and a self-equivalence $h: X \rightarrow X$ which induces the identity in homotopy groups but not in homology. For example, takke $X=\left(S^{n} \times S^{n}\right) \vee S^{2 n}$; let $h$ be the identity on $S^{n} \vee S^{n} \vee S^{2 n}$, and extend $h$ over the $2 n$-cell of $S^{n} \times S^{n}$ so as to have nontrivial degree on $S^{2 n}$. Then $h_{*}$ is not the identity in homology. But I assert that the inclusion map $S^{n} \vee S^{n} \vee S^{2 n} \subset X$ induces an epimorphism in homotopy groups, and so $h_{*}$ is the identity in homotopy groups. The proof of this assertion relies on the following:

Proposition 1.2. Let $f_{1}: Y_{1} \rightarrow Y_{1}^{\prime}$ and $f_{2}: Y_{2} \rightarrow Y_{2}^{\prime}$ be maps such that for $i=1,2, \Omega f_{i}: \Omega Y_{i} \rightarrow \Omega Y_{i}^{\prime}$ admits a coretraction $g_{i}: \Omega Y_{i}^{\prime} \rightarrow \Omega Y_{i}\left(\Omega f_{i} \circ g_{i} \simeq 1\right)$. Then $f_{1} \vee f_{2}: Y_{1} \vee Y_{2} \rightarrow Y_{1}^{\prime} \vee Y_{2}^{\prime}$ is onto in homotopy groups.

Proof. Utilize the fibration $\Omega Y_{1} * \Omega Y_{2} \rightarrow Y_{1} \vee Y_{2} \rightarrow Y_{1} \times Y_{2}$ to derive a split exact sequence in homotopy:

$$
\pi_{n}\left(\Omega Y_{1} * \Omega Y_{2}\right) \rightarrow \pi_{n}\left(Y_{1} \vee Y_{2}\right) \rightarrow \pi_{n}\left(Y_{1} \times Y_{2}\right)
$$

The splitting is defined by the inclusions $Y_{i} \subseteq Y_{1} \vee Y_{2}$. The sequence and the splitting are natural with respect to maps of $Y_{1}$ and $Y_{2}$. The hypotheses imply that $\Omega f_{1} * \Omega f_{2}: \Omega Y_{1} * \Omega Y_{2} \rightarrow \Omega Y_{1}^{\prime} * \Omega Y_{2}^{\prime}$ admits the coretraction $g_{1} * g_{2}$. It follows that $f_{1} \vee f_{2}$ is onto in homotopy as desired.

Apply the proposition with $Y_{1}=S^{n} \vee S^{n}, Y_{2}=S^{2 n}, Y_{1}^{\prime}=S^{n} \times S^{n}, Y_{2}^{\prime}$ $=Y_{2}, f_{1}=$ inclusion, $f_{2}=1_{S^{n}}$; the required coretraction for $\Omega f_{1}: \Omega\left(S^{n} \vee S^{n}\right)$ $\rightarrow \Omega\left(S^{n} \times S^{n}\right)=\Omega S^{n} \times \Omega S^{n}$ is obtained by multiplying together the two maps $\Omega S^{n} \rightarrow \Omega\left(S^{n} \vee S^{n}\right)$ induced by inclusions of $S^{n}$ into the wedge.

I conclude this section with a classification of homotopy types having universal cover homotopy equivalent to a given 1-connected space $X$ and $\pi_{1}$ isomorphic to a given group $G$.

First I shall describe a homotopy action of $\delta(X)$ on $B_{G(X)}$. The universal fibration $\zeta / B_{G(X)}$ comes equipped with a fixed equivalence $f_{\zeta}: X \rightarrow \zeta^{-1}(*)$. Given $[h] \in \mathcal{E}(X)$, consider the fibration $\zeta$ together with the equivalence 
$f_{5} \circ h: X \rightarrow \zeta^{-1}(*)$. As indicated by the remarks beginning this section, the space $B_{G(X)}$ classifies pairs $(\theta, f)$, where $\theta: E \rightarrow B$ is a fibration and $f$ : $X \rightarrow \theta^{-1}(*)$ is an equivalence. Therefore, associated to the pair $\left(\zeta, f_{\zeta} \circ h\right)$ there is a homotopy class of maps $\Gamma_{h}: B_{G(X)} \rightarrow B_{G(X)}$. We think of $\Gamma_{h}$ as the base map in a fibre homotopy equivalence which maps the universal fibration $\zeta$ so as to restrict to $h$ on the fibre $\zeta^{-1}(*)$. It is clear that these maps $\Gamma_{h}$ describe a homotopy action of $\delta(X)$ on $B_{G(X)}$.

Now let $\left[K(G, 1), B_{G(X)}\right]$ denote, as usual, the based homotopy classes of based maps. Then $\mathcal{E}(X)$ acts on the left via the homotopy action of $\mathcal{E}(X)$ on $B_{G(X)}$ just described. The automorphism group Aut $G$ acts on the right via composition with self-equivalences of $K(G, 1)$.

TheOREM 1.3. Let $X$ be a 1-connected space, $G$ a group. The homotopy types having universal cover homotopy equivalent to $X$ and $\pi_{1}$ isomorphic to $G$ are in 1-1 correspondence with the set .

$$
\mathcal{E}(X) \backslash\left[K(G, 1), B_{G(X)}\right] / \text { Aut } G .
$$

Proof. It will be convenient to work with triples of data, as follows. Suppose that $Y$ is a space, $\tilde{Y}$ its universal cover, $f: X \rightarrow \tilde{Y}$ a homotopy equivalence, and $\gamma: G \rightarrow \pi_{1}(Y)$ an isomorphism. I will associate to the triple $(Y, f, \gamma)$ an element of $\left[K(G, 1), B_{G(X)}\right]$. The isomorphism $\gamma$ defines a function denoted $\theta_{Y}: Y \rightarrow K(G, 1)$ inducing $\gamma^{-1}$ on $\pi_{1}$. As in the proof of Theorem 1.1, convert $\theta_{Y}$ into a fibration, denoted $\bar{\theta}_{Y}$. Utilize the homotopy equivalence $f$ to obtain a homotopy equivalence denoted $f_{Y}: X \rightarrow \bar{\theta}_{Y}^{-1}(*)$. The $X$-fibration $\left(\bar{\theta}_{Y}, f_{Y}\right)$ is classified by a map denoted $\varphi_{Y}: K(G, 1) \rightarrow B_{G(X)}$. Thus associated to the triple $(Y, f, \gamma)$ is the element $\varphi_{Y} \in\left[K(G, 1), B_{G(X)}\right]$.

Suppose $Y^{\prime}, f^{\prime}: X \rightarrow^{\approx} \tilde{Y}^{\prime}$, and $\gamma^{\prime}: G \approx \pi_{1}\left(Y^{\prime}\right)$ is another set of data and suppose that $j: Y \rightarrow Y^{\prime}$ is a homotopy equivalence. Then $j$ determines an automorphism $\beta=\gamma^{\prime-1} \circ j_{*} \circ \gamma$ of $G$ and a homotopy equivalence $h=$ $f^{\prime-1} \circ \tilde{j} \circ f$ of $X$. Here $\tilde{j}$ is the universal cover of $j$ and $f^{\prime-1}$ is a homotopy inverse of $f^{\prime}$. The diagram below is then homotopy commutative:

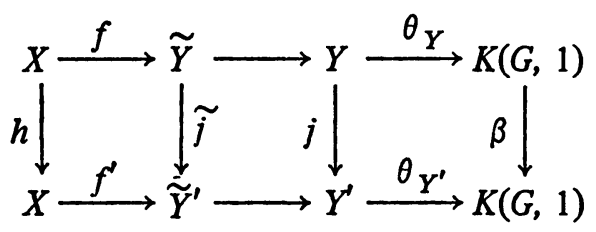

Let $\left(\bar{\theta}_{Y^{\prime}}, f_{Y^{\prime}}\right)$ denote the $X$-fibration obtained by converting the map $\theta_{Y^{\prime}}$ into a fibration, and using $f^{\prime}$ to identify $X$ and the fibre of $\bar{\theta}_{Y^{\prime}}$. The diagram (1.2) means that there exists a fibre homotopy equivalence $\bar{\theta}_{Y} \simeq \beta^{*} \bar{\theta}_{Y^{\prime}}$, which maps $X$ to itself by $h$. Therefore the diagram below is homotopy commutative: 


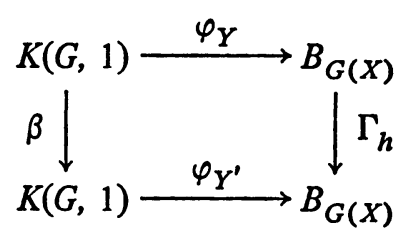

Here $\varphi_{Y}, \varphi_{Y^{\prime}}$ are the maps classifying $\left(\bar{\theta}_{Y}, f_{Y}\right)$ and $\left(\bar{\theta}_{Y^{\prime}}, f_{Y^{\prime}}\right)$, and $\Gamma_{h}$ is the action of $[h] \in \mathcal{E}(X)$ defined earlier. Therefore $\varphi_{Y^{\prime}} \simeq \Gamma_{h} \circ \varphi_{Y} \circ \beta^{-1}$ and so $\left[\varphi_{Y}\right]=\left[\varphi_{Y^{\prime}}\right]$ in $(1.1)$.

I have therefore defined a function which will be denoted $A$ mapping the set of homotopy types to be classified to the set (1.1). By following the converse portion of the proof of Theorem 1.1 one sees that $A$ is onto. On the other hand, suppose given $Y_{i}, f_{i}: X \approx \tilde{Y}_{i}, \gamma_{i}: G \approx \pi_{1}\left(Y_{i}\right)$, for $i=1,2$, and suppose that $A\left(Y_{1}\right)=A\left(Y_{2}\right)$. Then if $\varphi_{Y_{i}}: K(G, 1) \rightarrow B_{G(X)}, i=1,2$, denotes the map associated to the triple $\left(Y_{i}, f_{i}, \gamma_{i}\right)$, then $\left[\varphi_{Y_{1}}\right]=\left[\varphi_{Y_{2}}\right]$ and so there exist $\beta \in$ Aut $G$ and $h \in \mathcal{E}(X)$ such that

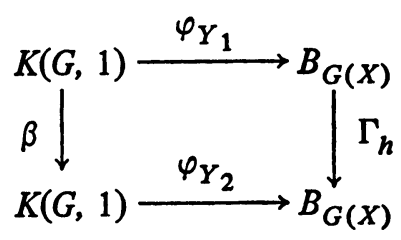

is homotopy. commutative. Therefore $\beta^{*} \varphi_{Y_{2}}^{*}(\zeta)$ is fibre homotopy equivalent to $\varphi_{Y_{1}}^{*}(\zeta)$. But $\varphi_{Y_{i}}$ classifies the fibration obtained from the map $\theta_{Y_{i}}: Y_{i} \rightarrow$ $K(G, 1)$, and so $\beta$ induces a homotopy equivalence $Y_{1} \approx Y_{2}$. This completes the proof of Theorem 1.3.

2. Homotopy actions on $h_{*}$-local spaces. In this section, I describe one fairly general situation where the obstructions (0.3) always vanish (Theorem 2.3). I then use this result to show that under certain conditions the invariant cohomology of a space with homotopy action can be realized as the cohomology of another space (Theorem 2.4). Theorem 2.5 is a result on the structure of $\mathcal{E}(X)$ when $X$ is $h_{*}$-local (cf. reference in Introduction).

Following Bousfield, a space $X$ is called $h_{*}$-local if any $h_{*}$-equivalence $f$ : $A \rightarrow B$ induces a bijection $f^{*}:[B, X] \rightarrow[A, X]$ of pointed homotopy classes of maps. Associated to any space $X$ is a space $E X$ and a map $\eta_{X}: X \rightarrow E X$ such that

(i) $\eta_{X}$ is an $h_{*}$-equivalence.

(ii) $E X$ is $h_{*}$-local.

The space $E X$ has as a result a well-defined homotopy type and is called the $h_{*}$-localization of $X$. Important special cases occur when $h_{*}=H_{*}(p ; R)$, where $R=\mathbf{Z} / p, p$ a prime, or $R$ is a subring of $Q$, the rational field. In case 
$X$ is 1-connected (or, more generally, nilpotent) then $E X$ coincides with the $R$-completion as defined in [4]. If in addition $R=\mathrm{Z} / p$ and $X$ is of finite type $\bmod p$, then $X \rightarrow E X$ coincides with the $p$-profinite completion as defined by Sullivan [8].

Given a generalized homology theory $h_{*}$ let $\tilde{P}$ denote the set of primes $p$ such that multiplication by $p$ is an isomorphism on $h_{*}(\mathrm{pt})$. Recall that a (possibly nonabelian) group $G$ is called uniquely $q$-divisible if the $q$ th power map $G \rightarrow G$ is a bijection.

Proposition 2.1. Let $X$ be connected and $h_{*}$-local. Assume $q \in \tilde{P}$. Then $\pi_{i}(X)$ is uniquely $q$-divisible.

Proof. The map $S^{i} \rightarrow S^{i}$ of degree $q$ induces multiplication by $q$ on $\tilde{h}_{*}\left(S^{i}\right)$, and is therefore an $h_{*}$-equivalence, since $q \in \tilde{P}$. Since $X$ is $h_{*}$-local the map of degree $q$ induces a bijection $\pi_{i}(X) \rightarrow \pi_{i}(X)$; the latter is multiplication by $q$ for $i \geqslant 2$, raising to the $q$ th power if $i=1$. Therefore $\pi_{i}(X)$ is uniquely $q$-divisible.

Suppose $X$ and $Y$ are connected, and that $v: X \rightarrow Y$ is a map. Then $v$ determines a vertex in the mapping space $\operatorname{hom}(X, Y)$. According to Bousfield [3], if $Y$ is $h_{*}$-local, so is each component of $\operatorname{hom}(X, Y)$. Therefore Proposition 2.1 implies

Corollary 2.2. If $Y$ is $h_{*}$-local, and $q \in \tilde{P}$, then $\pi_{i}(\operatorname{hom}(X, Y), v)$ is uniquely q-divisible for $i \geqslant 1$.

Now I come to the main theorem of this section.

ThEOREM 2.3. Let $G$ be a group of order $n$. Suppose that multiplication by $n$ is an isomorphism of $h_{*}(\mathrm{pt})$. Suppose that $X$ is $h_{*}$-local. Let $\alpha$ be a homotopy action of $G$ on $X$. Then the groups (0.3) are zero and so $\alpha$ is equivalent to a topological action of $G$ on some space $Y$.

Proof. We want to apply Theorem 1.1. The obstructions to replacing a homotopy action of $G$ on $X$ by a topological action reside in

$$
H^{i}\left(G ;\left\{\pi_{i-2}\left(G_{1}(X), 1\right)\right\}\right), \quad i \geqslant 3 .
$$

The space $G_{1}(X)$ is recognized as the component of $1_{X}$ in $\operatorname{hom}(X, X)$. The groups $\pi_{i-2}(\operatorname{hom}(X, X))$ are uniquely $q$-divisible (abelian) groups for all $q$ dividing $n$ by Corollary 2.2. Therefore the groups (2.1) are zero and the obstructions vanish.

Under the hypotheses of the above theorem, there is exactly one lifting in diagram (2), and therefore, the homotopy type of the quotient space obtained after replacing the homotopy action by a fixed point free topological action is well defined and may be denoted $X / \alpha$. 
Theorem 2.3 has two rather direct consequences, in the case where $h_{*}=$ $H_{*}(; R), R=\mathrm{Z} / p$ or $R \subseteq Q$. First there is a realizability theorem:

THEOREM 2.4. Let $X$ be any space, $G$ a group whose order is invertible in $R$, and $\alpha$ a homotopy action of $G$ on $X$. Then there exist a space $X_{\alpha}$ and a map $f$ : $X \rightarrow X_{\alpha}$ such that $f^{*}$ maps $h^{*}\left(X_{\alpha}\right)$ isomorphically onto $h^{*}(X)^{G}$, the cohomology of $X$ which is fixed by $G$.

Proof. The action $\alpha$ of $G$ on $X$ induces an action $E \alpha$ of $G$ on $E X$, where $E X$ denotes Bousfield's $h_{*}$-localization. The map $X \rightarrow E X$ is an $h_{*}$-equivalence. Since $E X$ is $h_{*}$-local and $|G|$ is invertible in $R$, Theorem 2.3 applies to show that $E \alpha$ is equivalent to a topological action of $G$ on some space $Y$. The latter may be assumed fixed point free. Set $X_{\alpha}=Y / G$. Since $|G|$ is invertible in $R$ and $Y \rightarrow X_{\alpha}$ is a regular covering with covering group $G, h^{*}\left(X_{\alpha}\right) \rightarrow$ $h^{*}(Y)$ maps isomorphically onto the $G$-invariant cohomology of $Y$. Composing with the $h_{*}$-equivalence $X \rightarrow E X \rightarrow Y$, one obtains a map $f$ satisfying the conclusion of the theorem.

Theorem 2.3 has an interesting application concerning the group $\mathcal{E}(X)$ when $X$ is $h_{*}$-local. Following Bousfield, let us informally define a group $G$ to be $h_{*}$-local if it occurs as a homotopy group of some $h_{*}$-local space. In general, if $X$ is $h_{*}$-local, $\mathcal{E}(X)$ is not necessarily $h_{*}$-local. Here is a simple example. Let $h_{*}=H_{*}(; \mathbf{Z} / p), p$ odd, and $X=K\left(\hat{\mathbf{Z}}_{p}, n\right)$, where $\hat{\mathbf{Z}}_{p}$ denotes the ring of $p$-adic integers. Then $\delta(X)$ is the group of units $\hat{\mathbf{Z}}_{p}^{*}$, which is isomorphic to $(\mathbf{Z} / p)^{*} \times \hat{\mathbf{Z}}_{p}$, since $p$ is odd, and so cannot be $h_{*}$-local, by Proposition 2.1. In this example, note that the subgroup $\hat{\mathbf{Z}}_{p}$, which of course is $h_{*}$-local, can be realized as the group of homotopy equivalences of $X$ which induce the identity on $h_{*}(X)$. Therefore one is led to conjecture that for any $h_{*}$-local space $X$, the kernel of the evaluation map $\mathcal{E}(X) \rightarrow$ Aut $h_{*}(X)$ is an $h_{*}$-local group. Let $\mathcal{E}_{1}(X) \subset \mathcal{E}(X)$ denote the above kernel. The following theorem supports the conjecture just stated.

THEOREM 2.5. Let $h_{*}=H_{*}(; R), R \subset Q$ or $R=\mathrm{Z} / p$, let $P$ denote the set of primes which are not invertible in $R$, and suppose $X$ is $h_{*}$-local. Then the only torsion occurring in the group $\mathcal{E}_{1}(X)$ is $P$-torsion.

Proof. Let $f: X \rightarrow X$ be a homotopy equivalence such that

(i) $f^{q} \simeq 1_{X}, q$ a prime, $q \notin P$,

(ii) $f_{*}=1: h_{*}(X) \rightarrow h_{*}(X)$.

Then by (i) $f$ defines a homotopy action of the cyclic group $G$ of order $q$ on $X$. Since $X$ is $h_{*}$-local and $q \in \tilde{P}$, Theorem 2.5 applies and we may assume that the action is a fixed point free topological action. Consider the covering space $X \rightarrow X / G$. This is a regular $q$-sheeted covering, the covering group acts trivially on $h_{*}(X)$, and $q$ is invertible in the coefficient ring $R$. It follows that 
the covering projection induces $h_{*}$-isomorphisms. Applying Bousfield's localization functor, we receive a homotopy equivalence $E X \rightarrow E(X / G)$. Since $X$ is $h_{*}$-local, the localization map $X \rightarrow E X$ is a homotopy equivalence. By examining the diagram

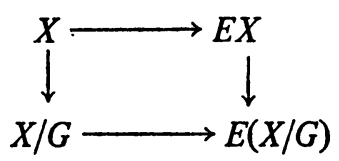

we see that the composition $X \rightarrow E(X / G)$ is a homotopy equivalence and so $X \rightarrow X / G$ has a left homotopy inverse. Therefore $f \simeq 1$.

Note. I do not know whether the result as stated is true for other generalized homology theories.

3. An example. In this section I present an example of a homotopy action which is not equivalent to a topological action. The group $G$ is $Z / 2$. It is convenient to take the following point of view. Let $X$ be a space, $f: X \rightarrow X$ a homotopy equivalence such that $f^{2} \simeq 1_{x}$. This defines a $\mathrm{Z} / 2$ homotopy action. If $f \simeq 1_{X}$, then the action is trivially equivalent to a topological action. So assume $f \not 1_{X}$. We represent $f$ and $1_{X}$ as points in their respective components of hom $(X, X)$. The situation is pictured as follows:
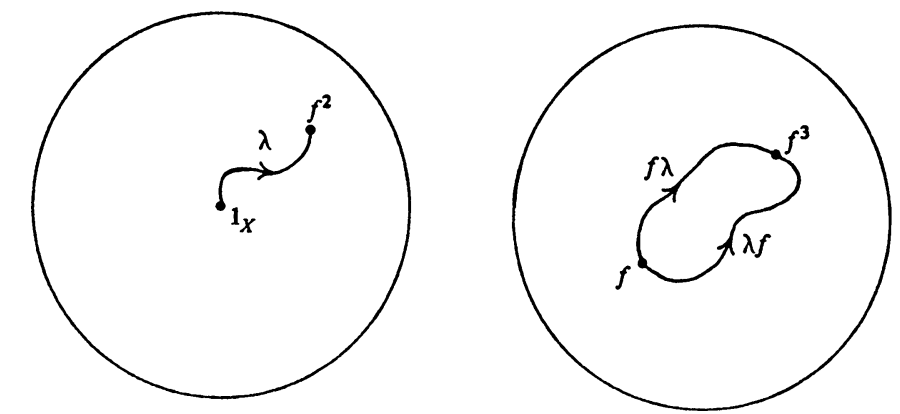

Since $f^{2} \simeq 1_{X}$, there is a path $\lambda$ joining $1_{X}$ to $f^{2}$. By composition we can define 2 paths, $\lambda f$ and $f \lambda$, connecting $f$ and $f^{3}$. The resulting loop represents an element $\theta$ of $\pi_{1}(\operatorname{hom}(X, X), f)$, which depends upon the choice of the path $\lambda$. If the given homotopy action of $\mathrm{Z} / 2$ is equivalent to another homotopy action of $\mathrm{Z} / 2$ on a space $Y$, utilizing a homotopy equivalence $h: X \rightarrow Y$, then the diagram (3.1) is equivalent to an analogous diagram for $Y$. (Make use of the equivalence $\operatorname{hom}(X, X) \rightarrow \operatorname{hom}(Y, Y)$ defined by $\varphi \rightarrow h \circ \varphi \circ h^{-1}$, where $h^{-1}$ denotes a homotopy inverse for $h$.) In the case of a topological action $f^{2} \simeq 1_{X}$; so, taking $\lambda$ to be the constant path, $\theta$ is the constant loop at $f$ and so is trivial. Therefore,

Proposition 3.1. If the given homotopy action of $\mathrm{Z} / 2$ on $X$ is equivalent to a 
topological action, then it is possible to choose $\lambda$ so that $\theta$ is trivial.

The present point of view is related to that of Theorem 1.1 as follows.

Represent $K(\mathrm{Z} / 2,1)$ as $R P^{\infty}$. Let $\alpha: \mathrm{Z} / 2 \rightarrow E(X)$ denote the given homotopy action, and consider the lifting problem of $\S 1$ :

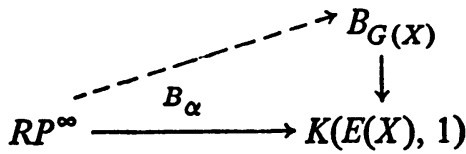

The path $\lambda$ is a specific realization of the fact that $f^{2} \simeq 1_{X}$; the choice of $\lambda$ determines a lifting of $B_{\alpha}$ over the 2-skeleton. It turns out that the element $\theta \in \pi_{1}(\operatorname{hom}(X, X), f)$ represents the obstruction to lifting $B_{\alpha}$ over the 3skeleton. Therefore, in the following example, where $\theta$ will be nontrivial for all choices of $\lambda$, there is no lift of $B_{\alpha}$ over the 3-skeleton of $R P^{\infty}$.

EXAMPLE. Assume $m>3$ and let $\alpha$ denote any element in the 2-primary component of $\pi_{n-1}\left(S^{m}\right)$, such that the order of $\alpha$ is divisible by 4 . Let

$$
X=\left(S^{m} \cup_{2 \alpha} e^{n}\right) \vee S_{1}^{n-1} \vee S_{2}^{n-1} \text {. }
$$

All maps and homotopies may be assumed pointed in the following discussion since $X$ is 2-connected. I introduce the notation:

(i) $i_{1}, i_{2}$ denote the functions $S^{n-1} \rightarrow X$ mapping $S^{n-1}$ with degree 1 on $S_{1}^{n-1}, S_{2}^{n-1}$, respectively.

(ii) $i: S^{m} \cup_{2 \alpha} e^{n} \subset X$ is the inclusion.

(iii) $p: S^{m} \cup_{2 \alpha} e^{n} \rightarrow S^{m} \cup_{2 \alpha} e^{n} \vee S^{n}$ pinches the boundary of a small $n$-ball in the cell $e^{n}$ to a point.

(iv) $\eta: S^{n} \rightarrow S^{n-1}$ represents the generator of $\pi_{n}\left(S^{n-1}\right) \approx Z / 2$.

Define a map $f: X \rightarrow X$ by stipulating:

(a) $f \mid S^{m} \cup_{2 \alpha} e^{n}=\left(i \bigvee\left(i_{2} \circ \eta\right)\right) \circ p$,

(b) $f \mid S_{1}^{n-1}$ represents $i \circ \alpha+i_{1}$,

(c) $f \mid S_{2}^{n-1}$ is the inclusion.

The map $f$ is thus obtained via the usual Puppe action of the homotopy groups of $X$ on the identity map. Now $\pi_{n-1}(X) \approx \pi_{n-1}\left(S^{m}\right) /(2 \alpha) \oplus \mathbf{Z} \oplus \mathbf{Z}$, so (b) implies that $f_{*} \neq$ id on $\pi_{n-1}(X)$ and so $f \neq 1_{X^{*}}$. On the other hand, since $i \circ \alpha$ and $\eta$ have order $2, f^{2}=f \circ f$ is homotopic to $1_{X}$. Therefore $f$ defines a homotopy action by $Z / 2$ on $X$. Let

$$
F: I \times X \rightarrow X
$$

denote a homotopy from $1_{X}$ to $f^{2}$. For the moment consider $F \mid S_{1}^{n-1} \times I$. This is a homotopy, which may be assumed pointed, from the inclusion of $S_{1}^{n-1}$ in $X$ to a map representing $i \circ 2 \alpha+i_{1}$ according to the construction of $f$. Therefore $F$ maps $S_{1}^{n-1} \times \dot{I}$ into the subspace $S^{m} \vee S_{1}^{n-1}$ of $X$, and so induces a map of pairs 


$$
\left(S_{1}^{n-1} \times I, S_{1}^{n-1} \times I \cup * \times I\right) \rightarrow\left(X, S^{m} \vee S_{1}^{n-1}\right)
$$

Since the order of $\alpha$ is divisible by $4,(3.4)$ has odd degree on the cell $e^{n}$. Associated to (3.4) is the map on quotient spaces

$$
S^{n} \stackrel{\bar{F}}{\rightarrow} S^{n} \vee S_{2}^{n-1} .
$$

If $i^{\prime}, i_{2}$ denote the inclusions of $S^{n}, S_{2}^{n-1}$ in the wedge, then, as an element of $\pi_{n}\left(S^{n} \vee S_{2}^{n-1}\right)$,

$$
\bar{F}=a \cdot i^{\prime}+b \cdot i_{2} \circ \eta
$$

where $a$ is odd and $b \in \mathrm{Z} / 2$.

According to the discussion at the start of this section, we check whether the diagram

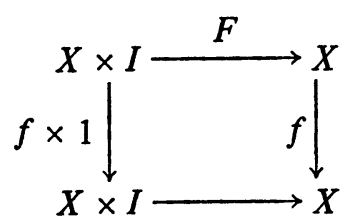

is homotopy commutative rel $X \times \dot{I}$. Precede the diagram by the inclusion $j$ : $S_{1}^{n-1} \times I \subset X \times I$, and follow the diagram by the map $q: X \rightarrow S^{n-1}$ which pinches $S^{m} \cup_{2 \alpha} e^{n}$ and $S_{1}^{n-1}$ to a point and has degree 1 on $S_{2}^{n-1}$. We then receive two functions,

$$
g_{1}=q \circ f \circ F \circ j, \quad g_{2}=q \circ F \circ(f \times 1) \circ j,
$$

mapping $S_{1}^{n-1} \times I$ to $S^{n-1}$. These agree on $S_{1}^{n-1} \times \dot{I}$. In fact, both map $S_{1}^{n-1} \times \dot{I}$ to the base point of $S^{n-1}$, and by further collapsing $* \times I$ define maps $\bar{g}_{1}, \bar{g}_{2}: S^{n} \rightarrow S^{n-1}$. In order for (3.7) to be homotopy commutative rel $X \times \dot{I}$, it is necessary that $g_{1} \simeq g_{2}$ rel $S_{1}^{n-1} \times \dot{I}$, and hence that $\bar{g}_{1} \approx \bar{g}_{2}$. But it is straightforward to check that since $a$ is odd in (3.6), in fact $\bar{g}_{1} \neq \bar{g}_{2}$. Consider $g_{1}$. The map $F \circ j$ is essentially (3.4). So we represent $g_{1}$ as a composition of maps of pairs

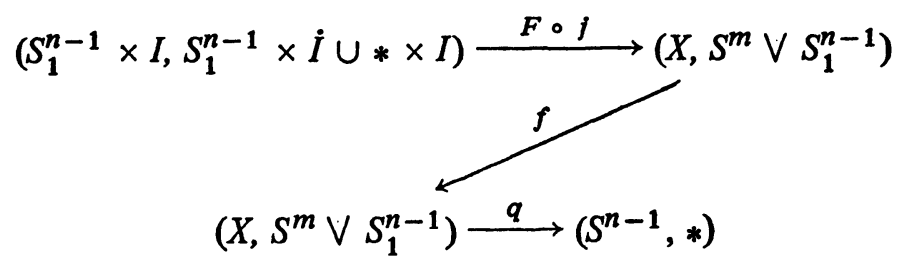

Representing $X /\left(S^{m} \vee S_{1}^{n-1}\right)$ as $S^{n} \vee S_{2}^{n-1}$, note that the map $\bar{f}: S^{n} \vee$ $S_{2}^{n-1} \rightarrow S^{n} \bigvee S_{2}^{n-1}$ induced by $f$ satisfies $\bar{f}_{*}\left(i^{\prime}\right)=i^{\prime}+i_{2} \circ \eta$. (Notation as in (3.6).) It follows that $\bar{g}_{1} \simeq(a+b) \eta$, where $a$ and $b$ are defined in (3.6). Next treat $g_{2}$ as the composition of the maps of pairs in the following diagram: 


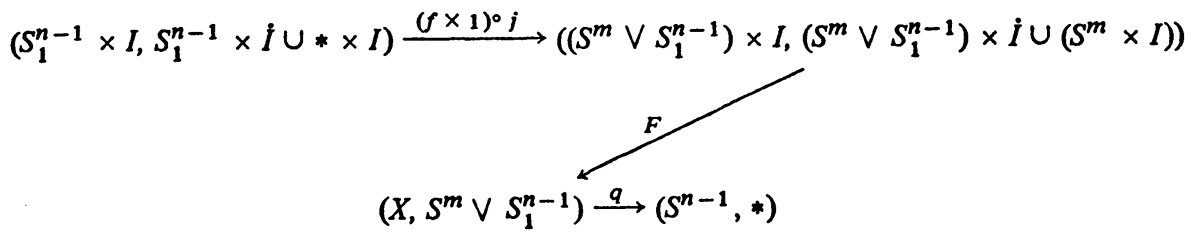

On quotient spaces the maps are

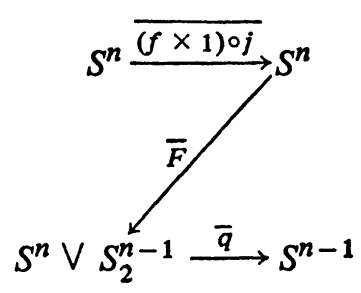

Now $\overline{(f \times 1)^{\circ} j}$ has degree $1, \bar{F}$ is given in (3.6), and $\bar{q}$ pinches $S^{n}$ to a point. It follows that

$$
\bar{g}_{2}=\bar{q} \circ \bar{F} \circ \overline{(f \times 1) \circ j}=b \cdot \eta \text {. }
$$

Therefore $\bar{g}_{1} \not \bar{g}_{2}$. Therefore there is no homotopy $F(3.3)$ for which the associated diagram (3.7) is homotopy commutative rel $X \times \dot{I}$. Therefore the elements $\theta$ defined at the start of this section are all nontrivial and so by Proposition 3.1 the given homotopy action by $\mathrm{Z} / 2$ is not equivalent to a topological action.

Appendix: Relative homotopy actions. Let $X$ be a space, which I will assume is a $\mathrm{CW}$ complex, and let $A$ be a subcomplex. Suppose a group $G$ acts topologically on $X$. If $A$ is $G$-invariant, then of course one obtains a topological action of $G$ on $A$. If $A$ is not $G$-invariant, then one might try to deform the $G$-action to make $A G$-invariant. It is difficult to work out a general theory which says when such deformation is possible. The same problem for homotopy actions is much simpler. It is useful to define the relative group $\mathcal{E}(X, A)$ of homotopy classes of homotopy equivalences of the pair $(X, A)$. Forgetting $A$ defines a homomorphism $\delta(X, A) \rightarrow^{\omega} \mathcal{E}(X)$, and the question is, when does a homotopy action of $G$ on $X$ factor through $\omega$ ? An affirmative answer is given in many situations by the following:

Proposition A.1. Suppose the pair $(X, A)$ is 2-connected and that $H^{i}\left(A ; \pi_{i}(X, A)\right)=H^{i-1}\left(A ; \pi_{i}(X, A)\right)=0$ for $i \geqslant 3$. Then $\omega: \mathcal{E}(X, A) \rightarrow$ $\mathcal{E}(X)$ is an isomorphism and so any homotopy action of a group $G$ on $X$ defines a homotopy action of $G$ on the pair $(X, A)$.

Proof. Let $f: X \rightarrow X$ be any continuous map. Consider the factorization problem pictured below: 


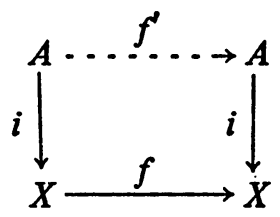

Here $i: A \subset X$ is the inclusion. The map $f^{\prime}$ is definable on the 2-skeleton of $A$ since $(X, A)$ is 2-connected. Obstructions to the existence of $f^{\prime}$ live in $H^{i}\left(A ; \pi_{i}(X, A)\right), i \geqslant 3$, and so are zero by hypothesis. Therefore $f^{\prime}$ exists so that (A.1) homotopy commutes. Now use the homotopy extension property to homotope $f$ to an extension of $f^{\prime}$.

Therefore, given $f$, there exists a homotopy from $f$ to a map of the pair $(X, A)$. Suppose two maps $f_{i}$ of $(X, A)$ are homotopic as maps of $X$. Then $f_{1} \mid A$ is homotopic in $X$ to $f_{2} \mid A$. Since $(X, A)$ is 2-connected, we may assume that this homotopy maps the 2-skeleton of $A \times I$ into $A$. Obstructions to deforming this homotopy into $A$ live in

$$
H^{i}\left(A \times I, A \times \dot{I} ; \pi_{i}(X, A)\right) \approx H^{i-1}\left(A ; \pi_{i}(X, A)\right),
$$

which is zero for $i \geqslant 3$ by hypothesis. Therefore the homotopy of $f_{1} \mid A$ and $f_{2} \mid A$ may be deformed into $A \operatorname{rel} A \times \dot{I}$. Utilize the homotopy extension property for the pair $(X \times I, X \times I \cup A \times I)$ to obtain a homotopy of the maps $f_{1}$ and $f_{2}$ as maps of pairs.

It follows that associated to each $[f] \in[X, X]$ is a unique $[\bar{f}] \in$ [( $X, A),(X, A)]$, which restricts to $[f]$ on $X$. It follows, using uniqueness, that if $[f] \in \mathcal{E}(X),[\bar{f}] \in \mathcal{E}(X, A)$. Therefore $\omega$ is an isomorphism.

For example, if $A$ is a skeleton of $X$, then, in general, one cannot restrict a homotopy action on $X$ to $A$, but if $X$ has a cell structure with no $(n+1)$-cells, and $A=X^{n}=X^{n+1}, n \geqslant 2$, then the conditions of the proposition hold for $(X, A)$ and any homotopy action on $X$ induces one on $(X, A)$ and hence, by restriction, a homotopy action on $A$.

Secondly, let us say that a based homotopy action takes values in $\delta(X, *)$, or, in the case of pairs, in $\mathcal{E}(X, A, *)$.

REMARK A.2. Let $\alpha: G \rightarrow \mathcal{E}(X, A, *)$ be a based homotopy action of $G$ on the pair $(X, A)$. Then $\alpha$ induces a homotopy action on the homotopy theoretic fibre of the inclusion $A \subset X$. For the fibre is realized as the space of paths in $X$ which start in $A$ and terminate in *. Given $g \in G$, and $\alpha(g) \in$ $\mathcal{E}(X, A, *)$, compose paths in $X$ with $\alpha(g)$.

Finally, suppose $\alpha: G \rightarrow \mathcal{E}(X, A)$ is a homotopy action of $G$ on the pair $(X, A)$; the action $\alpha$ is equivalent to a topological action on a pair $(Y, B)$ homotopy equivalent to $(X, A)$ if and only if a certain lifting problem analogous to $(0.2)$ has an affirmative solution. The lifting problem is stated in terms of the classifying space of the space $G(X, A)$ of all homotopy equivalenccs of the pair $(X, A)$. As in $\S 2$, if $X$ and $A$ are $h_{*}$-local, the component of 
the identity in $G(X, A)$ is $h_{*}$-local, and as a consequence there is:

Proposition A:3. Let $G$ be a group of order $n$. Suppose that multiplication by $n$ is an isomorphism of $h_{*}(\mathrm{pt})$. Suppose that $(X, A)$ is a pair of $h_{*}$-local spaces. Let $\alpha$ be a homotopy action of $G$ on $(X, A)$. Then $\alpha$ is equivalent to a topological action on some pair $(Y, B)$.

\section{REFERENCES}

1. J. F. Adams, A variant of E. H. Brown's representability theorem, Topology 10 (1971), 185-198.

2. Guy Allaud, On the classification of fibre spaces, Math. Z. 92 (1966), 110-125.

3. A. K. Bousfield, The localization of a space with respect to homology, Topology 14 (1975), 133-150.

4. A. K. Bousfield and D. M. Kan, Homotopy limits, completions, and localizations, Lecture Notes in Math., vol. 304, Springer-Verlag, Berlin and New York, 1972.

5. G. E. Cooke, Constructing spaces with interesting $Z_{p}$-cohomology via $\varepsilon$-actions on loop spaces, Cornell Univ. (preprint).

6. P. Hilton, Homotopy theory and duality, Gordon \& Breach, New York, 1965.

7. James D. Stasheff, A classification theorem for fibre spaces, Topology 2 (1963), 239-246.

8. D. Sullivan, Geometric topology, Part I: Localization, periodicity, and Galois symmetry, M.I.T. Press, Cambridge, Mass., 1970.

Department of Mathematics, Univerśtty of Maryland, College Park, Maryland 20742 